\title{
Towards Solving the Game of Go in One Dimension
}

by

\section{Isabel Christina Lewis}

\author{
A thesis submitted to \\ the Faculty of Graduate Studies and Research \\ in partial fulfillment of the requirements for the degree of \\ Master of Science
}

School of Mathematics and Statistics

Ottawa-Carleton Institute for Mathematics and Statistics

Carleton University

Ottawa, Ontario, Canada

23 August, 2007

(C) Copyright 2007

Isabel Christina Lewis 


$\begin{array}{ll}\begin{array}{l}\text { Library and } \\ \text { Archives Canada }\end{array} & \begin{array}{l}\text { Bibliothèque et } \\ \text { Archives Canada }\end{array} \\ \begin{array}{l}\text { Published Heritage } \\ \text { Branch }\end{array} & \begin{array}{l}\text { Direction du } \\ \text { Patrimoine de l'édition }\end{array} \\ \begin{array}{l}\text { 395 Wellington Street } \\ \text { Ottawa ON K1A ON4 }\end{array} & \begin{array}{l}\text { 395, rue Wellington } \\ \text { Ottawa ON K1A ON4 } \\ \text { Canada }\end{array}\end{array}$

Your file Votre référence ISBN: 978-0-494-33703-5 Our file Notre référence ISBN: 978-0-494-33703-5

NOTICE:

The author has granted a nonexclusive license allowing Library and Archives Canada to reproduce, publish, archive, preserve, conserve, communicate to the public by telecommunication or on the Internet, loan, distribute and sell theses worldwide, for commercial or noncommercial purposes, in microform, paper, electronic and/or any other formats.

The author retains copyright ownership and moral rights in this thesis. Neither the thesis nor substantial extracts from it may be printed or otherwise reproduced without the author's permission.
AVIS:

L'auteur a accordé une licence non exclusive permettant à la Bibliothèque et Archives Canada de reproduire, publier, archiver, sauvegarder, conserver, transmettre au public par télécommunication ou par l'Internet, prêter, distribuer et vendre des thèses partout dans le monde, à des fins commerciales ou autres, sur support microforme, papier, électronique et/ou autres formats.

L'auteur conserve la propriété du droit d'auteur et des droits moraux qui protège cette thèse. $\mathrm{Ni}$ la thèse ni des extraits substantiels de celle-ci ne doivent être imprimés ou autrement reproduits sans son autorisation.
In compliance with the Canadian

Privacy Act some supporting forms may have been removed from this thesis.

While these forms may be included in the document page count, their removal does not represent any loss of content from the thesis.
Conformément à la loi canadienne sur la protection de la vie privée, quelques formulaires secondaires ont été enlevés de cette thèse.

Bien que ces formulaires aient inclus dans la pagination, il n'y aura aucun contenu manquant.

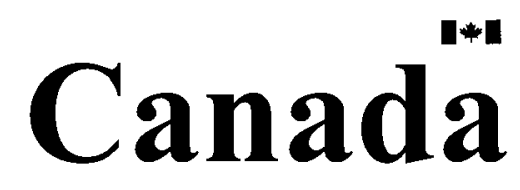




\begin{abstract}
The analysis in this paper concerns a game called One-dimensional Go. We describe and refine the rules of this game in Chapter 1.

One-dimensional Go is a game of perfect information between two players who alternate moves and use no random devices. Such games are known as combinatorial games. The foundation of combinatorial game theory was laid down in Conway's On Numbers and Games and Berlekamp, Conway and Guy's Winning Ways, and is described in Chapter 2.

In Chapter 3, the theory is used to determine an optimal strategy for both players and applied to the general case.
\end{abstract}




\section{Acknowledgements}

I wish to express my sincere appreciation to my thesis supervisor Dr Brett Stevens for his guidance, suggestions and support throughout the course of this research.

I am indebted to Drs Michael Albert, Richard Nowakowski and David Wolfe, for the manuscript of their book, Lessons in Play: an Introduction to the Combinatorial Theory of Games,

I am grateful to Dr John Poland for his advice and encouragement and to Dr Steven Wang for his valuable comments.

Specifically the author appreciates Dr Paul-Eugène Parent, Dr Pat Morin, Dr Zhicheng Gao and Dr Benjamin Steinberg for acting as examining committee.

I wish to convey my love and thanks to my family for their support and understanding - my daughters Naomi and Chloe, my mother Mary van Embden, and especially my husband David, to whom I dedicate this thesis. 


\section{Contents}

$\begin{array}{ll}\text { Abstract } & \text { ii }\end{array}$

Acknowledgements $\quad$ iii

Contents iv

$\begin{array}{ll}\text { Introduction } & 1\end{array}$

1 Playing One-dimensional Go 2

1.1 Notation . . . . . . . . . . . . . . . . . . . 4

1.2 Capturing Stones . . . . . . . . . . . . . . . . . . 5

1.3 The Rule of Ko . . . . . . . . . . . . . . 6

1.4 Establishing Territory . . . . . . . . . . . . 8

2 Combinatorial Game Theory 10

2.1 Outcome Classes . . . . . . . . . . . . . . . . 10

2.2 Infinitesimals and Switches . . . . . . . . . . . 12

2.3 Game Sums . . . . . . . . . . . . . . . . 13

2.4 Canonical Form . . . . . . . . . . . . . . . . 14

2.5 Stops . . . . . . . . . . . . . . . 16

iv 
3 Analysis 18

3.1 Values of Games . . . . . . . . . . . . . 18

3.2 Strategy . . . . . . . . . . . . . . 26

3.3 Ending the Game . . . . . . . . . . . . . . . . . . . 34

4 Conclusion $\quad 37$

A Tables $\quad 43$

B Recursion $\quad 51$

$\begin{array}{ll}\text { Bibliography } & 54\end{array}$ 


\section{Introduction}

The ancient game of Go originated in China more than 4000 yeas ago. The modern game has a number of local dialects. The Chinese, Japanese, North American and New Zealand versions are most commonly played in professional matches, and there are other less well known variations.

The game is usually played on a grid of $19 \times 19$ intersecting lines, although other sizes of board are possible. Beginners often use a $9 \times 9$ or $13 \times 13$ board. The two players are designated as Black and White. They take turns placing a stone of their own colour on one of the intersections between the lines. Their objective is to enclose territory and to capture the opponent's stones while avoiding capture of their own stones.

This paper seeks to find a general solution to a one-dimensional version of the classic game, using the conventions of the North American game of Go as closely as possible. In order to facilitate the analysis we made a number of simplifying assumptions, which are explained in the text. Further work may lead to a full solution. 


\section{Chapter 1}

\section{Playing One-dimensional Go}

In this one-dimensional game, the 'board' consists of a single straight line with $n$ nodes at equal intervals, including at the two ends of the line. The two players take turns placing a stone on a vacant node. One player places black stones and the other places white stones. Black plays first. Two or more stones of the same colour placed on consecutive nodes form a group.

The game ends when both players pass on consecutive moves. At the end of the game the players count one point for each vacant node inside their own territory, and one point for each prisoner captured. A player's territory consists of any number of vacant nodes with a stone of her own colour adjacent to each end. If the territory includes an end of the board, it is adjacent to only one of the player's stones.

To capture an opponent's stone, or group of stones, a player places a stone of her own colour on all vacant nodes adjacent to it. A vacant node adjacent to a stone is called a liberty. Unlike the two-dimensional game, each single played stone and group of stones has two liberties, unless it abuts an end of 
the board when it has one liberty. If a player increases the size of a group, the number of its liberties does not increase. In fact, if a player extends a group to include an end of the board, the number of liberties decreases from two to one. When capturing a group, a player scores one point as prisoner for each stone in the captured group.

As in the regular game of Go, once a stone is played it can not be moved. If it is captured by the opposing player, it is removed from the board and the node is now vacant.

Either player may choose to pass at their turn and, if the other player wishes to play another move, the game continues.

A player is not permitted to

- commit suicide

- play a stone in a position which returns the configuration of the board to one which is identical to a previous one (rule of $k o$ ).

The 'no suicide' rule means that it is illegal for a player to place a stone so that it has no liberties or creates a group with no liberties, unless the move results in the immediate capture of the opponent's stone or group of stones.

The rule of $k o$ is intended to prevent a repetition of the same set of moves in an endless loop. It can also be used by a player as a strategic device to divert her opponent from defending from an attack elsewhere on the board. In order to simplify the analysis, we have evaluated the $k o$ in isolation from the rest of the board. This simplified version of the rule of $k o$ is explained in section 1.3 .

The Chinese rules are also often used in North America. Here the players 
earn a point for each of their own stones left on the board after play has ended. The effect of using this rule in One-dimensional Go is discussed in the Conclusion and shown in Table 4.1.

\subsection{Notation}

During play, the board consists of a number of blocks of consecutive vacant nodes between two played stones. There are six possible types of block since either end of the block may be adjacent to a black stone, a white stone or an end of the board. A block of $k$ vacant nodes is denoted

- $b w(k)$ when its adjacent stones are opposite colours

- $b b(k)$ when its adjacent stones are both black

- $w w(k)$ when its adjacent stones are both white

- $b d(k)$ when it has one adjacent black stone and an end of the board

- $w d(k)$ when it has one adjacent white stone and an end of the board

- $d d(k)$ when it includes both the ends of the board, i.e. this denotes the initial board before play starts.

The notation $b b(0)$ describes two black stones on consecutive nodes. The nodes themselves are numbered 0 to $n-1$ from left to right. Thus the configuration of the entire board can be described. Figure 1.1 shows a board of size 15 with configuration $b d(1), b b(2), b w(4), w w(1), w d(3)$. It has black stones at position 1 and 4 and white stones at position 9 and 11 . 


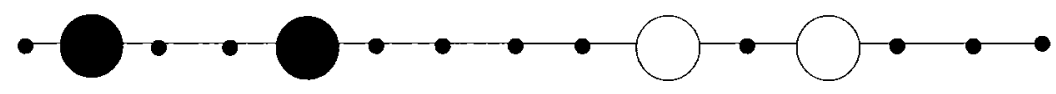

Figure 1.1: A sample One-dimensional Go game.

\subsection{Capturing Stones}

This paper will use the usual convention of describing Black as 'she' and White as 'he' [3].

To capture an opponent's stone, or group of stones, a player places a stone on every liberty adjacent to that stone or group. To avoid being captured, a player avoids leaving a stone or group with only one liberty. When Black's move creates $b w(0)$ or $b d(0)$ she risks a capture by White, which results in his gaining a prisoner as well as territory. Similarly, a move by White which creates $b w(0)$ or $w d(0)$ risks a capture by Black. Therefore, it is suboptimal to play in these positions unless it is part of a larger strategy which could result in an overall gain.

Lemma 1.1. For board size $n \geq 3$, it is suboptimal for a player to place a stone on the end of the board when the first three nodes are all vacant.

Proof. Suppose positions 0,1 and 2 are vacant and Black plays at 0 creating $b d(0)$. Then White plays adjacent to her stone on the only liberty, captures the black stone and creates $w d(1)$. By the 'no suicide' rule, Black can not play on the end node again. So this is now White's territory, giving him an advantage of 2 points. By the same reasoning, if positions $n-1, n-2, n-3$ are vacant and Black plays at $n-1$, White can capture the stone and gain 2 points. 

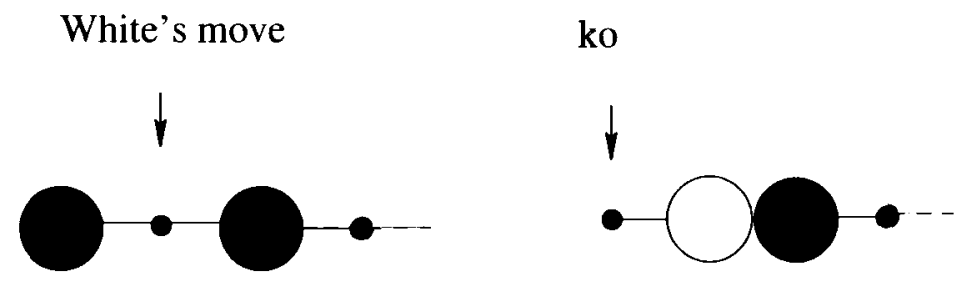

Figure 1.2: An example of the ko rule.

Similarly, if White plays at 0 or $n-1$ creating $w d(0)$, Black gains 2 points.

\subsection{The Rule of Ko}

When the players can repeat the same configuration in an infinite loop by alternately capturing and recapturing the same stone, the play is known as a $k o$. In order to avoid creating such a loop, the player whose stone has been captured is not permitted to recapture on the next move. In other words, she must make at least one other move elsewhere on the board before returning to the same position. In Figure 1.2, black stones are placed at positions 0 and 2 and White moves to capture the stone at the end of the board. The ko at position 0 prevents Black from recapturing on her next move. So, in this example, the white stone is now threatening her stone at position 2.

Lemma 1.2. For board size $n>5$, it is suboptimal for Black to place a stone on the end of the board when the move results in the configuration $b d(0), b b(1)$ and then at least one vacant node.

Proof. Case 1. Positions 0, 1 and 3 are vacant and Black has an existing stone in position 2. Black plays at 0 creating $b d(0)$, and White plays at 1 
capturing the stone. This is a ko, as shown in Figure 1.2. Since Black must now play elsewhere, White may capture the black stone in position 2 on his next move for a total of 4 points.

Case 2. Position 0 and 3 are vacant, Black has an existing stone in position 2 and White has an existing stone in position 1. Black plays at 0 capturing the white stone and creating $b d(0)$. Now there is a $k o$ is at position 1, so White can not recapture immediately. However, the configuration of the board is the same as shown in Figure 1.2, and White can take advantage of it after his next move for net gain of 3 points.

Similarly, if White places a stone on the end of the board and the move results in the configuration $w d(0), w w(1)$ and then at least one vacant node, Black will gain an advantage of at least 3 points. As with Lemma 1.1, Lemma 1.2 applies to either end of the board.

If a player has an opportunity to force her opponent to play at the end of the board, she will try to do this. Therefore, a player should guard against giving his opponent the chance to do so.

Corollary 1.3. It is suboptimal to play at 2 or $n-3$ when the four end nodes of the board are all vacant

Proof. Black plays at 2 (or $n-3$ ) creating $b d(2)$, and White responds by placing a stone at position 1 (or $n-2$ ). Black has two possible options:

- play elsewhere on the board allowing White to capture the stone, as well as gaining two points for territory

- capture White's stone by placing a stone at the end of the board, violating Lemma 1.2. 


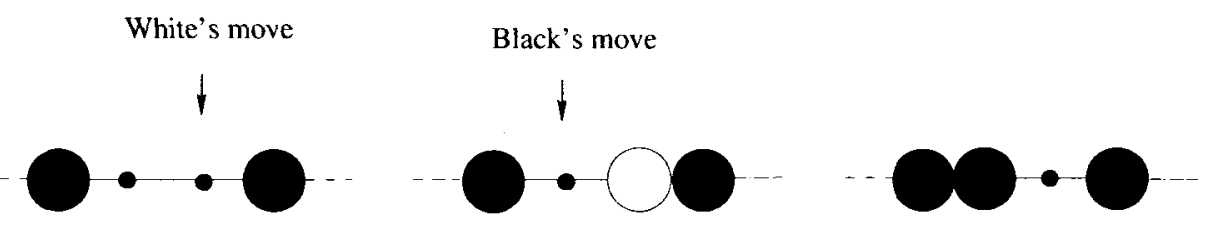

Figure 1.3: An example of Black territory.

Similarly, Black gains the advantage if White creates $w d(2)$.

By the same argument, a player can attempt to force her opponent to play at position 2. The general value of $b d(2 i)$ is analyzed in detail in Chapter 3.

\subsection{Establishing Territory}

In the usual two-dimensional version of Go, a player builds an 'army' of stones to capture the opponent's stones or to establish territory. However, in this one-dimensional game only two stones are needed to attack an opposing stone or group of stones, or to defend a territory. When an end of the board is involved a single stone may be used.

Because of the 'no suicide' rule, White can never play in the blocks $b d(1)$ or $b b(1)$, and these blocks are established as Black's territory. Similarly, Black can not play in $w d(1)$ or $w w(1)$ and these are White's territory.

If White tries to play in a block $b b(2)$, he can only place a stone adjacent to a black stone creating $b w(0)$. Black can immediately retaliate by capturing the white stone, and will do so in order to defend her boundary. Although her territory is reduced by one point, the captured white prisoner gives her one more point and White has not gained any advantage, as can be 
seen in Figure 1.3. Since Black is no worse off than she was before White's move, this is known as a reversible move. This important concept will be explained more fully in the next chapter. Thus the blocks $b b(2)$ and $w w(2)$ are completely defendable and are also considered to be Black and White territory respectively.

It is suboptimal for either player to play into a block $b w(1)$, since this would create a group of stones with only one liberty. This is equivalent to seki in the traditional Go game. While either player may play into a block $b w(2)$, they gain nothing by doing so and it is equivalent to dame [3]. Both $b w(1)$ and $b w(2)$ are neutral territory. Thus all play takes place in blocks that are not established as Black, White or neutral territory. 


\section{Chapter 2}

\section{Combinatorial Game Theory}

Go is an example of a game of perfect information between two players. In order to analyze the game and find each player's optimal strategy, every move can be given a value. These values are dependent on all possible options available to both the player and the opponent. Some values are numerical, but often they are not numbers in the traditional sense. Combinatorial game theory was developed to facilitate this type of analysis.

\section{$2.1 \quad$ Outcome Classes}

Combinatorial game theory deals with games of pure strategy played with no random devices such as dice or cards. A game $G$ is defined by the options available to each of the players. The two players are termed Left and Right. They play alternately until one of them has no legal move and at that point the other player wins the game. $G^{L}$ and $G^{R}$ are the set of options available to Left and Right respectively. This is all the information needed to completely 
determine a game. Thus we denote $G=\left\{G^{L} \mid G^{R}\right\}$.

If Left moves first, then either Left can force a win moving first or Right can force a win playing second, but not both [1]. And if Right moves first, then either Right can force a win moving first or Left can force a win playing second, but not both. As a result, a game can be categorized according to one of four possible outcome classes, as shown in the following table.

\begin{tabular}{|l|l|l|}
\hline Class & Name & Definition \\
\hline $\mathcal{L}$ & positive & Left can force a win moving 1st or 2 nd \\
\hline $\mathcal{R}$ & negative & Right can force a win moving 1st or 2nd \\
\hline $\mathcal{N}$ & fuzzy & the Next (1st) player can force a win \\
\hline $\mathcal{P}$ & zero & the Previous (2nd) player can force a win \\
\hline
\end{tabular}

The outcome of a game is related to its value, as we will explain. These values are determined using a set of axioms first identified by John Conway $[5]$.

If $G^{L}$ and $G^{R}$ are both empty sets, then $G=\{\mid\}=0$. In this case neither player has a legal move, and therefore the first player loses and $G$ is in class $\mathcal{P}$. If $G^{L}$ is empty and $G^{R}$ is the set $\{0\}$, then $G=\{\mid 0\}=-1$. Now only Right has a legal move and $G$ is in class $\mathcal{R}$. Similarly, $G=\{0 \mid\}=1$ is in class $\mathcal{L}$.

Using these first three numbers, $\{\mid-1\}=-2,\{-1 \mid 0\}=-\frac{1}{2},\{0 \mid 1\}=\frac{1}{2}$ and $\{1 \mid\}=2$ can be constructed. If $G^{L}$ and $G^{R}$ are both numbers and $G^{L}<$ $G^{R}$, then $G=\left\{G^{L} \mid G^{R}\right\}$ is also a number. All numbers can be constructed in this way. They form a field and include real numbers, transfinite numbers and infinitesimals [5]. If $j$ is an infinitesimal, then $j<x$ for all positive numbers $x$. All the integers and the dyadic rational numbers are built in a 
finite number of steps. The rest of the reals appear in $\omega$ steps. These have sometimes been termed surreal numbers [6] and are also used in nonstandard analysis [5].

\section{$2.2 \quad$ Infinitesimals and Switches}

So some games are also numbers. However, games are not restricted to numbers. Even when both $G^{L}$ and $G^{R}$ are numbers, game $G=\left\{G^{L} \mid G^{R}\right\}$ is not a number when $G^{L} \geq G^{R}$.

Firstly, consider the case when $G^{L}=G^{R}$. For example, when either player can make a move but scores zero. Here $G=\{0 \mid 0\}$ and is denoted by $*$. The next player to move wins the game, and $*$ is the fuzzy class $\mathcal{N}$ [5]. The game $*$ is termed an infinitesimal and, by definition, $*<x$ for all positive numbers $x$. It is said to be incomparable with zero, denoted $* \| 0$. For two games $G$ and $H, G \| H$ if $G$ is neither greater than, less than nor equal to $H[1]$. In general, if $g$ is a number, then $\{g \mid g\}=g *$, which can be viewed as the addition of $*$ to a game of value $g$. In other words, the value of game $\{g \mid g\}$ is infinitesimally close to $g$.

The games up and down, defined as $\uparrow=\{0 \mid *\}$ and $\downarrow=\{* \mid 0\}$ respectively, are also examples of infinitesimals. There are other infinitesimals defined in combinatorial game theory, but these will not be used in our analysis.

Secondly, when $G^{L}>G^{R}$ the game is called a switch. The position $\{y \mid z\}$, where $y$ and $z$ are numbers and $y>z$, can be rewritten as $\{y \mid z\}=\frac{y+z}{2} \pm \frac{y-z}{2}$, where $\pm \frac{y-z}{2}$ is shorthand for $\left\{\frac{y-z}{2} \mid-\frac{y-z}{2}\right\}$. Such games are termed hot games, indicating that both players have an advantage in playing next and would 
like the opportunity to do so. Such situations arise frequently when playing One-dimensional Go.

\subsection{Game Sums}

As play proceeds, many games split into independent components each of which is a game in itself. To understand the game as a whole, it can be analyzed as the sum of these component games. For example, the game of NIM is a collection of heaps and it has been solved using this property [4]. The endgame of standard two-dimensional Go itself has been extensively studied and the players' strategies improved using these methods [3]. The game of One-dimensional Go also splits into separate playing regions and can readily be described as the sum of its component games.

Using Conway's definitions [5], games form a commutative group with addition

$$
G+H=\left\{G^{L}+H, G+H^{L} \mid G^{R}+H, G+H^{R}\right\}
$$

Inverse is defined as

$$
-G=\left\{-G^{R} \mid-G^{L}\right\}
$$

and identity as

$$
0=\{\mid\}
$$

Let $G$ and $H$ be games where $G=\left\{G^{L} \mid G^{R}\right\}$ and $H=h$ is a number. Then $G+H=\left\{G^{L}+h \mid G^{R}+h\right\}$. Also, $G+*=\left\{G^{L}+* \mid G^{R}+*\right\}$. The game $\{0 \mid 0\}=*$ is its own inverse, and $*+*=\{* \mid *\}=0$. 
By convention, all values in a game are shown from Left's point of view. In some sense, the value of a game $G$ to Right is $-G$, since any gain to Left can be viewed as a loss to Right and vice versa. Suppose $G=\{3 \mid-1\}$. This means that the value of game $G$ to Left is 3 when Left plays first, and -1 when Right plays first. $-G=\{1 \mid-3\}$ are the gains to Right.

\subsection{Canonical Form}

Conway also defines equality and inequality [1]. Let $G$ and $H$ be games. Then

$$
\begin{gathered}
G=H \text { if } \forall X, G+X \text { has the same outcome as } H+X \\
G \geq H \text { if } \forall X, \text { Left wins } G+X \text { whenever Left wins } H+X \\
G \leq H \text { if } \forall X, \text { Right wins } G+X \text { whenever Right wins } H+X
\end{gathered}
$$

These are equivalent to $G-H=0, G-H \geq 0$ and $G-H \leq 0$ respectively.

Thus two different games may be equal. Every game $G$ has a unique simplest form which is equal to it, called $G$ 's canonical form. When adding the values of two or more component games, the sum is simplified and written in canonical form. There are only two simplifications needed to achicve this.

1. Remove dominated options

2. Bypass reversible options

Let $A$ and $B$ be two of Left's options. If $A \geq B$, then $A$ is said to dominate $B$. Left would not play $B$ if $A$ is available, therefore $B$ can be 


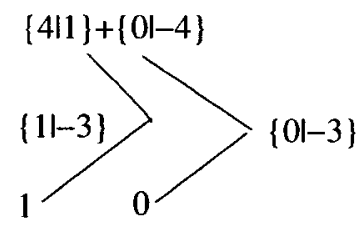

Figure 2.1: Left wins playing second.

removed. Similarly, for two of Right's options $C$ and $D$, then $C$ dominates $D$ if $C \leq D$ and $D$ can be removed.

The following example illustrates the removal of dominated options from a game sum. Let $G=\{3 \mid 0\}$ and $H=\{1 \mid-3\}$. Then $G+H=$ $\{\{4 \mid 0\},\{4 \mid 1\} \mid\{1 \mid-3\},\{0 \mid-3\}\}$. To show that Left's option $A=\{4 \mid 1\}$ dominates Left's option $B=\{4 \mid 0\}$, we need to show that $A \geq B \Leftrightarrow A-B \geq 0$. In other words, that Left wins playing second in game $A-B$. Figure 2.1 shows $A-B=A+(-B)=\{4 \mid 1\}+\{0 \mid-4\}$. Right moves first in one of two possible options; $1+\{0 \mid-4\}=\{1 \mid-3\}$ or $-4+\{4 \mid 1\}=\{0 \mid-3\}$. Then Left moving second results in either $1(\operatorname{class} \mathcal{L})$ or $0($ class $\mathcal{P})$. Whichever option Right chooses, Left wins playing second. Therefore for Left, $\{4 \mid 1\}$ dominates $\{4 \mid 0\}$. By a similar reasoning, for Right $\{0 \mid-3\}$ dominates $\{1 \mid-3\}$. In canonical form, $G+H=\{\{4 \mid 1\} \mid\{0 \mid-3\}\}$.

A reversible move by Left is one to which Right can make an immediate response which leaves him in a position at least as good as the one before Left's move. Lemma 1.1 is an example of a reversible option in Onedimensional Go. If Black plays at position 0, White immediately plays at position 1, capturing the black stone and gaining one point for territory. Thus Black's move at 0 is said to reverse to White's move at 1 with a score of -2 . Figure 1.3 illustrates an example of a reversible move by White. 


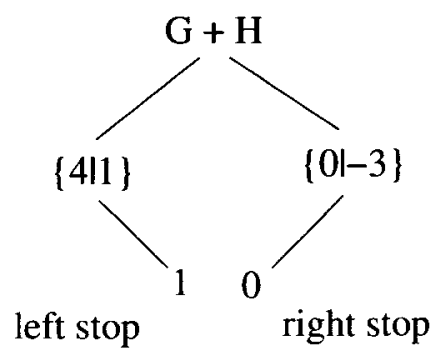

Figure 2.2: Determining the stops of a game.

\subsection{Stops}

After a certain number of moves, the value of a game will become a number.

The number arrived at when Left moves first, is called the left stop. Similarly, if Right moves first the number is called the right stop.

In the example in the previous section, $G=\{3 \mid 0\}, H=\{1 \mid-3\}$ and $G+H=\{\{4 \mid 1\} \mid\{0 \mid-3\}\}$. If Left moves first, she can choose to move in either $G$ or $H$. Since the two players alternate moves, Right will move in the other component of the game. So, if Left moves first in $G$ and then Right moves in $H$, the value to Left is $3+(-3)=0$. On the other hand, if she moves first in $H$ and then Right moves in $G$, the value is $1+0=1$. Therefore, Left will prefer to play $H$ first. If Right plays first he also prefers to move in $H$, forcing Left to play in $G$. The left stop of game $G+H$ is 1 , and the right stop is 0 . Figure 4.1 shows the stops as the leaves of the game tree when the game is written in canonical form.

For game $G$, denote the left stop and right stop by $\mathbf{L S}(G)$ and $\operatorname{RS}(G)$ respectively [1]. If $G$ is a number then $\mathbf{L S}(G)=\mathbf{R S}(G)=G$. Otherwise, the stops are defined in a mutually recursive manner; 


$$
\begin{aligned}
& \mathbf{L S}(G)=\max \mathbf{R S}\left(G^{L}\right) \\
& \mathbf{R S}(G)=\min \mathbf{L S}\left(G^{R}\right)
\end{aligned}
$$

In the simple example above, it is easy to calculate the canonical form and, hence, the stops. However, as the number of possible moves open to each player increases, the number of calculations needed to determine the canonical form increases exponentially. 


\section{Chapter 3}

\section{Analysis}

At any time during play, the game of One-dimensional Go can be viewed as the sum of its component games. These components are the blocks described in Chapter 1. The work involved in finding the canonical form of these sums, as well as the left and right stops, was accomplished using an open source program named the Combinatorial Game Suite [8]. This useful tool can also be used to program recursions and was indispensable for this research.

\subsection{Values of Games}

Black is always the left player. Suppose she is to move next and places a stone inside a block $b w(k)$. She can place her stone $m$ nodes from her own existing stone, where $0 \leq m \leq k-1$. This move creates two new blocks; $b b(m)$ and $b w(k-m-1)$. The value to Black of a move in $b w(k)$ is the set of sums of the values of the two new blocks. This is denoted $b w(k)^{L}=\{b b(m)+b w(k-$ $m-1) \mid 0 \leq m \leq k-1\}$. There are $k$ values to be considered. However, since 
the sum will always be shown in canonical form, some of these values will be removed. If White moves in $b w(k)$ he creates blocks $w w(m)$ and $b w(k-m-1)$ and, therefore, $b w(k)^{R}=\{(w w(m)+b w(k-m-1) \mid 0 \leq m \leq k-1\}$.

The block $b w(k)$ has the same value to both players since each views it as $k$ vacant nodes adjacent to a stone of their own colour at one end and a stone of their opponent's colour at the other. Thus the values to White are the negatives of the values to Black, and $b w(k)^{R}=-b w(k)^{L}$.

Placing a stone into $b w(1)$ would create a group with only one liberty, so neither player chooses to play there. Thus $b w(1)$ is seki and $b w(1)=0$. Either player may play into a block $b w(2)$ by placing a stone adjacent to their own colour for a score of 0 . In the standard game of Go such a move is called dame [3]. Thus $b w(2)=\{0 \mid 0\}=*$, an infinitesimal. The block $b w(2)$ is known as an impartial game since the options for both players are the same. The values of such games are always infinitesimals.

Both players avoid creating the block $b w(0)$, since their stone would be left with only one liberty, and would be immediately captured. A capture of a black stone by White is denoted by $\bullet$, and has a value of -1 . A capture of a white stone by Black is denoted by 0 , and has a value of 1 .

Similarly, Black can place her stone in a block $b b(k)$ creating $b b(m)$ and $b b(k-m-1)$. When playing in $w w(k)$, she creates $b w(m)$ and $b w(k-m-1)$. Thus $b b(k)^{L}=\{b b(m)+b b(k-m-1) \mid 0 \leq m \leq k-1\}$ and $w w(k)^{L}=$ $\{b w(m)+b w(k-m-1) \mid 0 \leq m \leq k-1\}$. From White's point of view, $b b(k)^{R}=-w w(k)^{L}$ and $w w(k)^{R}=-b b(k)^{L}$. The values of $b b(k)$ and $w w(k)$ can be calculated recursively starting from $k=0, k=1$ and $k=2 . b b(0)$ is a group of two black stones on consecutive nodes, and has a value of $0, b b(1)$ 
is one point of black territory and has a value of 1 , and $b b(2)$ is two points of black territory with value 2 , as can be seen in Figure 1.3. By the same reasoning, $w w(0)=0, w w(1)=-1$ and $w w(2)=-2$.

The other values for the games $b w(k), b b(k)$ and $w w(k)$ are found recursively. For example, if Black moves in $b w(3)$ she has three possible moves;

1. $b b(0)+b w(2)=0+*=*$

2. $b b(1)+b w(1)=1+0=1$

3. $b b(2)+b w(0)$, which reverses to

$$
b w(1)+w w(1)+\bullet=0+(-1)+(-1)=-2 .
$$

Since Black plays to maximize her score, her move 2 dominates the other two options, and is called Black's dominant move. White also has three options, which are the negatives of Black's options. He plays to minimize Black's score. Therefore, $b w(3)=\{*, 1,-2 \mid *,-1,2\}$. Simplifying this to canonical form, $b w(3)=\{1 \mid-1\}$ or \pm 1 .

In general, if Black moves in the block $b w(k), k \geq 3$, to create $b b(k-1)$ and $b w(0)$, the move reverses to $b w(k-2)+w w(1)+\bullet=b w(k-2)-2$. Similarly, if Black moves in the block $w w(k), k \geq 3$, to create $b w(k-1)$ and $b w(0)$, the move reverses to $w w(k-2)+w w(1)+\bullet=w w(k-2)-2$. These are always suboptimal moves for Black. Thus, such a move is dominated by Black's other options, and is removed. By the same reasoning, it is suboptimal for White to create $b w(0)$ inside a block $b w(k)$ or $b b(k)$. This also shows that the boundaries of the blocks $b w(k), b b(k)$ and $w w(k)$ are protected and a move by either player breaks these blocks into a sum of games. 
The form of the games $b w(k), b b(k)$ and $w w(k)$ becomes very complicated for quite small values of $k$, as shown in Table A.6 at the end of the paper. For example, $b w(9)= \pm(\{\{5 \mid 3\} \mid \pm 1\},\{\{5 \mid 2 *\} \mid 0\}\})$ and $b b(12)=\{\{\{\{8 \mid 6\} \mid\{5 * \mid 3 *\}\} \mid 2\} \mid \pm(1 *)\}$. These values are calculated using the program Cgsuite [8], which uses a simplified notation: $b w(9)=$ $\pm(\{5|3| \mid \pm 1\},\{5|2 *| \mid 0\})$ and $b b(12)=\{8|6||5 *| 3 *|||2||| \mid \pm(1 *)\}$. In this simplified notation the order of precedence is shown by the number of vertical bars, with a single bar having the least precedence.

The remaining types of blocks, $b d(k), w d(k)$ and $d d(k)$ can now be evaluated. The initial board of $n$ vacant nodes is denoted $d d(n)$ and, by definition, Black places a stone first. Although this is the only time this game is played, for the sake of consistency the recursion is written as $d d(k)^{L}=\{b d(m)+b d(k-m-1) \mid 0 \leq m \leq k-1\}$ and $d d(k)^{R}=$ $\{w d(m)+w d(k-m-1) \mid 0 \leq m \geq k-1 . \quad d d(0)$ would describe a nonexistent board and has no value. $d d(1)$ is a degenerate case where neither player has a legal move since the single node has no liberties. $d d(2)$ describes a board consisting of only the end nodes. Whichever player moves first will be immediately captured, so both players would choose to pass and the game ends with no score. However, all other values of $d d(k)$ can be found from $b d(k)$ and $w d(k)$, so the recursion depends on initializing these values.

By the same reasoning as before, $b d(k)^{L}=\{b d(m)+b b(k-m-1) \mid 0 \leq$ $m \leq k-1\}$ and $b d(k)^{R}=\{w d(m)+b w(k-m-1) \mid 0 \leq m \leq k-1\}$, and $w d(k)=-b d(k)$. As described in Chapter $1, b d(1)$ is a point of black territory and has a value of 1 . Similarly, $w d(1)=-1$. By Lemma 1.1, $b d(0)$ reverses to $(w d(1)+\bullet)$ with a value of -2 , and $w d(0)$ reverses to $(b d(1)+0)$ with a 
value of 2 . These values assume that the there are at least three vacant nodes at the end of the board when the reversible move is made. By Corollary 1.3 $b d(2)$ and $w d(2)$ are also reversible moves, and $w d(2)=-b d(2)$. However, evaluating $b d(2)$ is not at all straightforward.

Corollary 1.3 shows that Black has two possible options when White attacks her stone at position 2. If she plays elsewhere on the board and permits the capture, White gains nodes 0 and 2 as territory as well as a prisoner for a total of -3 . White could gain more if Black's stone at position 2 is part of a group. On the other hand, if Black captures White's stone she is forced to place her stone on the end node. Although White cannot recapture on the next move because of the $k o$ at position 1 , he can do so after both players have moved elsewhere. Now he not only gains the end node as territory, but his stone threatens Black's stone at position 2, as can be seen in Figure 1.2. Since this time the $k o$ prevents Black from recapturing, White still gains nodes 0 and 2 as territory. He also captures two black prisoners to the one white prisoner already captured by Black. So White still has a net gain of at least -3 . If Black can play into $b d(2)$ first, her best move is to place a stone at position 1 preventing the attack as well as gaining her one point for $b d(1)$. So $b d(2)=\{1 \mid-3\}$ is used for the recursion. However, this is a simplified version of the value since it does not include every possible option for each player.

The black stones at positions 0 and 2 are called dead stones, indicating that they are destined for capture. White does not need capture them immediately, but can leave them on the board until the end of the game so that he is free to play elsewhere in the meantime. However, the capture of 
the black stone at position 2 changes the configuration of the block adjacent to it. When it changes $b w(k)$ to $w w(k-1)$, or $b b(k)$ to $b w(k-1)$, White usually gains at least one extra point. Thus the exact gain to White could be greater than the value used in the recursion.

A further difficulty arises from the fact that one of Black's options when playing into $d d(4)$ is to play at position 2 , creating $b d(1)$ and $b d(2)$. In this case, White cannot attack at position 1 , since the ko prevents recapture and there is no other legal move on the board for him. This observation shows that $d d(4)= \pm 3$, which can be directly initialized.

Unfortunately, fixing the value of $b d(2)$ is not sufficient to run the recursion successfully, because now a similar problem occurs at $b d(4)$. Again, White can attack by placing a stone adjacent to the black stone and Black has two options. If she allows the capture, White gains one prisoner, one point for territory in $w w(1)$, and leaves $w d(3)$ to be played. The net score is $-1+(-1)+\{1 \mid-2\}=\{-1 \mid-4\}$. If Black captures the attacking white stone, she is forced into creating $b d(2)$. This time White can attack on two consecutive moves, resulting in the loss of two white prisoners, before Black is forced to the end of the board and the ko prevents immediate recapture. If there is room on the board for White to move elsewhere, he will move to capture the three dead black stones in positions 0,2 and 4 at the end of the game. At the same time he will also gain three points for $w d(1), w w(1)$, $w w(1)$ for a net gain of -4 points. Each of these captures is in a $k o$, and Black can not recapture provided that White makes them on his next three consecutive moves.

If Black can move first, she can place a stone at position 1 to create 
$b d(1)+b b(2)=1+2=3$. So $b d(4)=\{3 \mid-4,\{-1 \mid-4\}\}$, provided that White has room on the board to make a legal move after the ko capture at position 0 . Again, this is a simplified version of $b d(4)$. When $n=6$, White has no legal move when the ko prevents him from recapturing the black stone at position 0 . In this case, to initiate such an attack would be a suboptimal move for him and $b d(4)=\{3 \mid-4,\{-1 \mid-4\}\}$ can not be used in the recursion to evaluate $d d(6)$. Since Black has only three possible options, $d d(6)=*$ can be calculated, and initialized.

In general, for $n>2 i+3$, if White attacks in $b d(2 i)$ by creating $w d(2 i-1)$, and Black responds by capturing at each subsequent attack, White will gain $-(i+1)$ points for territory and capture $i+1$ black prisoners to $i$ white prisoners lost to Black. So White threatens to gain $-(i+2)$ points by this move. If Black does not respond, White gains -2 points by capturing the black stone at position $2 i+1$ and creates $w d(2 i-1)$, while Black gains one extra move.

Nevertheless, the move which creates a block $b d(2 i)$ is not reversible for every value of $i$. Suppose White attacks on $i$ consecutive moves with Black capturing $i$ prisoners. At the end of this exchange there is a dead black stone at position 0. When White moves to capture it, Black can place a stone at position 3 between her stones at positions 2 and 4, creating a group of 3 black stones. White can capture the entire group on his next move, but this is not a ko and Black can retaliate by capturing the attacking white stone immediately. So by sacrificing one more stone, Black can prevent White from taking further prisoners. White has gained a total of four prisoners as well as one point of territory. Black recaptures the white stone for a total of $i+1$ 
prisoners, as well as saving her remaining stones and $i-2$ points for territory. The net result is $2 i-6$ points and the block $b w(2)=*$.

The move to $b d(2 i)$ is reversible if White can make an immediate response which leaves him in a position which is at least as good as before Black's move. Therefore $b d(2 i)$ is not reversible for $i \geq 4$. For $i=3$, White can make the attacking move in block $b d(6)$ for a score of $*$, so $b d(6)^{R}=*$. However, this value may not be exact. When White makes his initial attack, Black has the option of allowing the capture and playing elsewhere. White gains -2 points and creates the block $w d(5)=\{2|0||-1|-3\}$, which is usually to his advantage. However, it does not take the value of Black's extra move into account, and this depends on the configuration of the rest of the board. As before, the value of the block $d d(8)$ must be calculated separately.

In summary, Black's move to $b d(2 i)$ is reversible for $0 \leq i \leq 3$, provided that there is a legal move for White elsewhere on the board after the ko capture at position 0 . This depends on the configuration of the rest of the board when Black makes the move creating $b d(2 i)$. The simplified values given above assume that the block $b d(2 i)$ is created by Black on her opening move in $d d(n)$. If $b d(2 i)$ is created after other stones have already been placed on the board it is not necessarily reversible. If Black's stone at position $2 i$ is part of a group, then White can gain more points. By the same argument, creating $w d(i), 0 \leq i \leq 3$ is a reversible move by White if there is a legal move for Black elsewhere on the board after the ko capture at position 0 . 


\section{$3.2 \quad$ Strategy}

Once the values of the different blocks have been calculated, each player's dominant move, or set of moves, can be found for every case. This is accomplished using the left and right stops. For example, $b w(3)=\{1 \mid-1\}$ as shown in the previous section. Therefore, $\mathbf{L S}(b w(3))=1$ and $\mathbf{R S}(b w(3))=-1$. If Black moves first, she has three possible moves, only one of which gives her a score of $1: b b(1)+b w(1)$. So this is her dominant move in this game. It is possible for a player to have more than one option resulting in the optimal score, but no option can give the players a better score than the stops. Any option resulting in a score which is less than the left stop for Black (or more than the right stop for White) is suboptimal.

The values of the stops may not be immediately obvious from the value of the game. For example, $b b(12)=\{8|6||5 *| 3 *|||2||| \mid \pm(1 *)\}$, showing that $b b(12)^{L}=\{8|6||5 *| 3 *|| \mid 2\}$ and $b b(12)^{R}=\{ \pm(1 *)\}=\{1 * \mid-1 *\}$. Thus $\mathbf{L S}(b b(12))=2$ and $\mathbf{R S}(b b(12))=1$, since the right stop of $1 *$ is 1 .

Once the stops have been calculated, the dominant moves for each player can be found. In the example above, $b b(12)^{L}=\{b b(m)+b b(11-m) \mid 0 \leq m \leq$ $11\}$. For every value of $m$, the right stop of the game $(b b(m)+b b(11-m))$ is calculated and those which result in a value of 2 are Black's dominant moves. Similarly, the dominant moves by White are found when the left stop of the game $(b w(m)+b w(11-m))$ equals 1 . Because of the symmetry of the blocks $b b(k)$, only the values $0 \leq m \leq 5$ need be considered. These are calculated using the program Cgsuite [8], and are shown in Table 3.1. The dominant moves for Black are at $m=2,3,4$ or 5 , and the dominant moves for White are at $m=1,2,3$ or 5 . 


\begin{tabular}{|c|c|c|}
\hline$m$ & $\mathbf{R S}(b b(m)+b b(11-m))$ & $\mathbf{L S}(b w(m)+b w(11-m))$ \\
\hline 0 & 0 & \\
\hline 1 & 1 & 1 \\
\hline 2 & 2 & 1 \\
\hline 3 & 2 & 1 \\
\hline 4 & 2 & 2 \\
\hline 5 & 2 & 1 \\
\hline
\end{tabular}

Table 3.1: The stops for game $b b(12)$.

Note that the block $b w(0)$ is omitted from the recursion, since it causes an infinite loop. This occurs because the $k o$ rule can not be written into the program. However, as shown in the previous section, creating the block $b w(0)$ is a reversible move and is suboptimal for that player. In this case, $b w(0)+b w(11)$ reverses to $b b(1)+b b(10)+\circ=\{9|6 *\|4 \mid\| 2 *\}$. Therefore, $\mathbf{L S}(b w(0)+b w(11))=4$.

Black's dominant moves for each type of block are shown in the tables at the end of the paper. They can be used to determine a winning strategy for Black.

Table A.2 shows Black's dominant moves in $b w(k)$ for $3 \leq k \leq 18$. An optimal strategy for Black is to play at $m=2$ creating $b b(2)=2$ for all values of $k$ except $k=3,7$ or 8 . As shown previously, Black's dominant move for $b w(3)$ is to play at $m=1$. The table also shows Black's dominant move in $b w(7)$ is at $m=3$. For $b w(8)$, Black has dominant moves at $m=4$ and $m=6$. Since $b w(k)^{R}=-b w(k)^{L}$, White can use the same strategy and 
create the block $w w(2)$ for all values of $k$ except $k=3,7$ or 8 .

Since Black's move in creating $b b(2)$ gains her an immediate score of 2 points, she will prefer any move which gives her more than 2 points. Table A.1 shows the left stops for all blocks with $1 \leq k \leq 32$. When $\mathbf{L S}(b w(k))-\mathbf{R S}(b w(k))=\mathbf{L S}(b w(k))+\mathbf{L S}(b w(k))>2$, Black's optimal strategy is to play immediately into $b w(k)$ if she has the opportunity. $b w(4)=$ $\{2 \mid-2\}= \pm 2$ and $b w(5)=\{2 * \mid-2 *\}= \pm(2 *)$. Black gains an advantage of 4 points by moving first in either of these blocks. By the same argument, White also gains an advantage of -4 points by moving in these blocks first. These are examples of hot games. So the optimal strategy for both players is to avoid creating the blocks $b w(4)$ or $b w(5)$, which is the reason why moving at $m=2$ is not an optimal strategy when playing in $b w(7)$ or $b w(8)$.

Table A.3 shows Black's dominant moves in $b b(k)$ for $3 \leq k \leq 32$. For all odd values of $k$ except for $k=3$ or 13, Black's optimal strategy again is to play at $m=2$ creating $b b(2)=2$. For $b b(3)$, Black's dominant moves are to play at either $m=1$, creating $b b(1)+b b(1)=2$, or at $m=0$ creating $b b(0)+b b(2)=2$. For $b b(13)$, Black's only dominant move is at $m=6$. $\boldsymbol{R S}(b b(2)+b b(k-3))=2$ for even $k \geq 6$. However, this is not always the dominant move for Black. The table shows that $\mathbf{L S}(b b(k))=3$ for $k=14$, $22,24,28,30$. The values of $b b(k), k>32$ have not been calculated.

It should be noted that creating any block $b b(k)$ implies a previous suboptimal move by Black since White can gain an advantage by moving first. The options $b b(k)^{R}=-w w(k)^{L}$, and the left stops for $w w(k)$ are shown in Table A.4. In this case Black's strategy is clear. For all odd values of $k$, Black's only dominant move is at $m=\frac{k-1}{2}$. For even values of $k$, Black 
may have a set of several dominant moves, which always includes $m=\frac{k-2}{2}$. White has the same options when he moves first in $b b(k)$. Particularly for odd values of $k \geq 9, \mathbf{L S}(b b(k))=3$ and $\mathbf{R S}(b b(k))=0$ and White has an advantage of 3 points by moving next after Black has created $b b(k)$.

When $\mathbf{L S}(b b(k))-\mathbf{R S}(b b(k))=\mathbf{L S}(b b(k))+\mathbf{L S}(w w(k))>2, b b(k)$ is a hot game and White's optimal strategy is to play immediately into $b b(k)$ if he can. $(b b(4)=\{3 \mid *\}$ gives White an advantage of 3 points, and $(b b(5)=\{4 \mid 0\}$ gives White an advantage of 4 points. By the same argument, Black's strategy is to move in a block $w w(k)$ if she can. Again, $w w(5)=\{0 \mid-4\}$ and Black can deprive White of 4 points by moving first.

Table A.5 shows Black's dominant moves in $b d(k), 3 \leq k \leq 24$. It can be seen that there is a dominant move for Black at $m=1$ for $3 \leq k \leq 8$ and at $m=k-3$ for $k \geq 8$. Since $b d(k)^{L}=\{b d(m)+b b(k-m-1) \mid 0 \leq m \leq k-1\}$, playing at $m=k-3$ is equivalent to creating the blocks $b d(k-3)$ and $b b(2)=2$.

As explained previously, the blocks $b d(2), b d(4)$ and $b d(6)$ are reversible provided that White has a legal move somewhere on the board after the $k o$ capture at position 0 . If the configuration of the board before Black creates the block $b d(k)$ does not allow this, $b d(2), b d(4)$ and $b d(6)$ are not reversible. In those circumstances $b d(2)=2, b d(4)=\{3 \mid-1 *\}$ and $b d(6)=$ $\{\{4 \mid 1\},\{4 \mid 1 *\} \mid\{1 \mid-3\}\}$.

Creating the reversible block $b d(2)$ or $b d(4)$ is a suboptimal move for Black and this option will be dominated and removed. So using a simplified value will not change the outcome. However, creating the block $b d(6)$ can sometimes be a dominant move, as can be seen in Table A.5. Indeed, it is 
Black's only dominant move for $\mathbf{L S}(b d(9))=2$. The simplified value of the reversible block $b d(6)$ may not be exact, as was explained in the previous section. The same uncertainty applies to the value of $\mathbf{R S}(w d(6))$.

The game $b d(3)=\{2 \mid-1\}$, so $\mathbf{L S}(b d(3))-\mathbf{R S}(b d(3))=3>2$. This is another example of a hot game where White can gain an advantage by moving next and Black avoids creating such a block.

Creating the block $w d(2 i), 0 \leq i \leq 3$ is a reversible move by White, and Black's dominant move is to to play at position $2 i$ threatening a capture. For all other blocks $w d(k)$ Black has a dominant move at $m=1$ for every value of $k$ except for $k=7$, where her dominant moves are at $m=3$ or 5 . This is because a Black move at $m=1$ in $w d(7)$ creates the hot game $b w(5)$, where White can play immediately for an advantage of 4 points. Similarly, White's strategy for the non-reversible blocks $b d(k)$, is to move at $m=1$ and create $w d(1)$, except for $b d(7)$ where his dominant moves are at $m=3$ or 5 .

For the blocks $d d(k)$ Black has a dominant move at $m=1$ for every value of $k \geq 3$. Black's options on her opening move are $d d(k)^{L}=d d(n)^{L}=$ $\{(b d(m)+b d(n-m-1)), 0 \leq m \leq n-1\}$. When $n$ is even, either $m$ is even or $n-m-1$ is even, therefore moving at $m=1$ may be the only dominant opening move for Black. When $n$ and $m$ are both odd, $n-m-1$ is also odd, so any odd value of $m$ avoids the reversible Black move which creates $b d(2 i)$, $0 \leq i \leq 3$. It may be observed that in the classic game of Go, a typical board consists of an odd number of nodes.

At any time during the game, the only moves by Black which result in an immediate gain in territory to her are the creation of the blocks $b b(2)=2$, $b b(1)=1$ or $b d(1)=1$. One of these is a dominant move unless it also 
creates a hot game which would give White an immediate advantage, or the previous play gives Black the opportunity to play in a block where the left stop has a greater value. White gains most points for territory by creating $w w(2)=-2, w w(1)=-1$ or $w d(1)=-1$.

Given an existing block, the stops are calculated on the assumption that both players make a dominant move on each turn thereafter. If White makes a suboptimal move, it means that he chooses an option which is not one of his dominant moves. In other words, he creates a new game which has a right stop which is larger than the right stop of the game into which he has just played. As long as Black replies with any move which is one of her dominant moves in the new game, she will increase her score. If Black makes a suboptimal move, she creates a new game with a left stop which is less than the left stop of the game she has just played into, and White can improve his score. These suboptimal moves often involve the creation of a hot game into which the opposing player can make an immediate gain on the next move. In particular, Black gains extra points by playing in the blocks $b w(4), b w(5)$, $w d(3), w w(4), w w(5)$ and $w w(2 i+1), i \geq 4$. The blocks $w w(3)=\{0 \mid-2\}$ and $b w(3)= \pm 1$ are also hot games which give Black an advantage of 2 points when she plays first. She can also respond immediately to the blocks $b w(0)$ and $w d(2 i), 0 \leq i \leq 3$ when they are created by a reversible move by White. White has similar options to improve his score when Black makes a suboptimal move.

Tables A.2, A.3 and A.5 show that creating the block $b b(0)$ is never a dominant move for Black and, by implication, creating the block $w w(0)$ is never a dominant move for White. In other words, in One-dimensional Go 


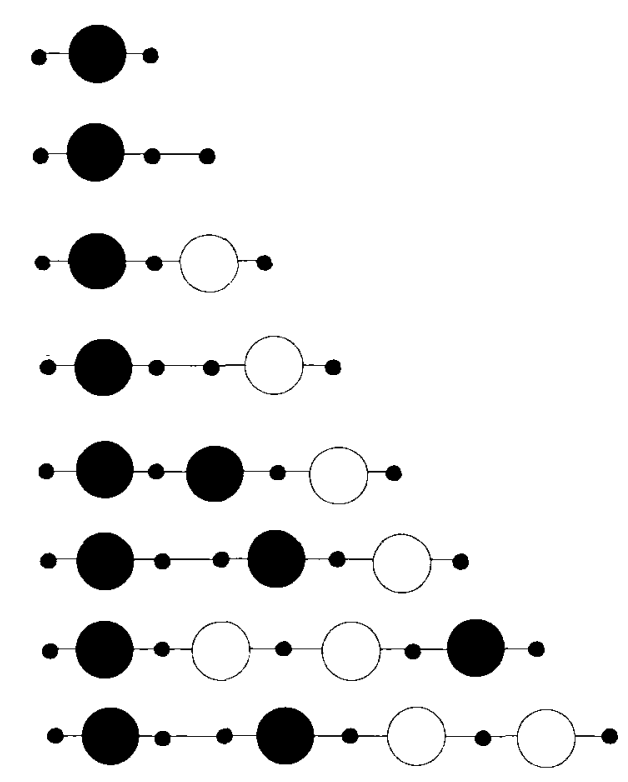

Figure 3.1: The final board for $3 \leq n \leq 10$.

it is always suboptimal for a player to create a group. This is a fundamental difference from the standard two-dimensional game. It also justifies using the simplified version of Lemma 1.1 to evaluate $b d(0)=-2$.

In summary, then, Black's strategy is to open at node 1 or $n-2$ creating $b d(1)=1$. For $n=3$ or 4 this move ends the game. Otherwise, White's optimal response is to create $w d(1)=-1$ at the opposite end of the board, except when $n=9$. In this case, Black's opening move creates $b d(1)$ and $b d(7)$, to which White's reply is either at $m=3$ or 5 . For all other values of $n \geq 5$, the board configuration is now $b d(1), b w(n-4), w d(1)$ and Black has the next move. For $k \geq 4$ Black has a dominant move in $b w(k)$ at $m=2$, except for $k=7$ or 8 . Figure 3.1 shows the final board for $3 \leq n \leq 10$.

For $n \geq 11$ the board configuration is now $b d(1), b b(2), b w(n-7), w d(1)$ 


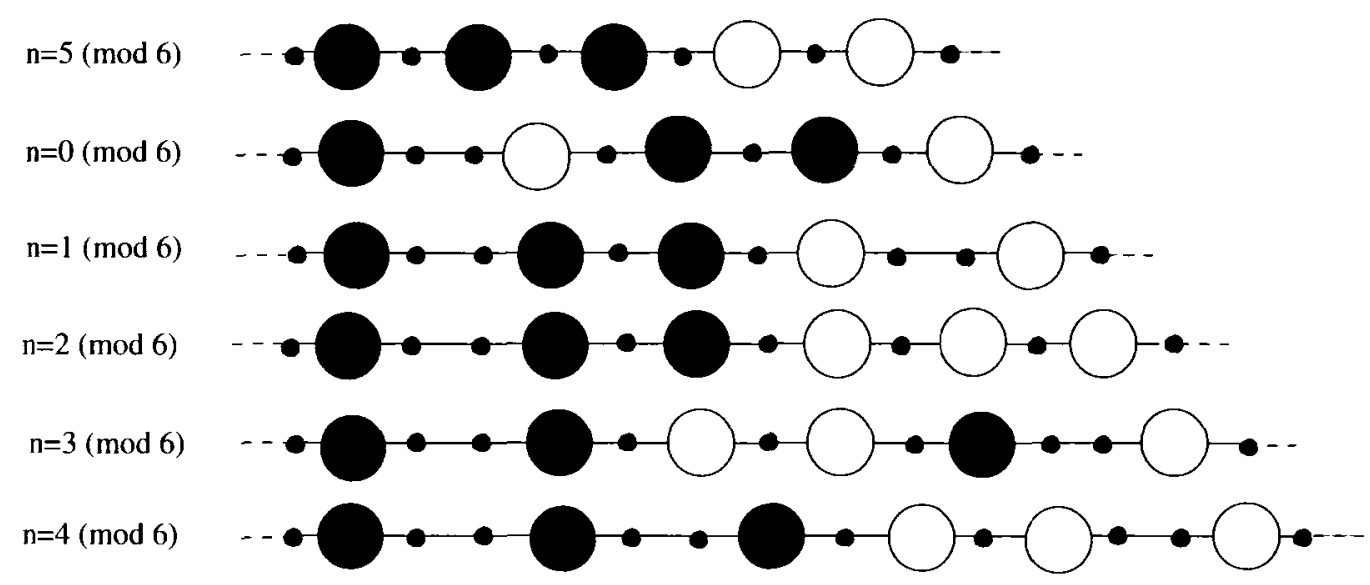

Figure 3.2: The middle section of the final board for $n \geq 11$.

and White has the next move. White also has a dominant move in $b w(k)$, $k \geq 4$, at $m=2$, except for $k=7$ or 8 . The two players can alternate these two moves $l$ times until the only unplayed block is $b w(k), 7 \leq k \leq 12$, in the middle of the board and Black is to play next. Up to now, Black has scored $(2 l-1)$ points for territory to White's score of $-(2 l-1)$. In other words, the two players are tied and $k=(n-6 l+2)$.

If $k=7$ Black moves at $m=3$ and if $k=8$ Black moves at $m=4$ or 6. Any of these moves results in a net score of 1 point to Black. Otherwise, Black moves at $m=2$ gaining 2 points for territory and it is White's turn to move in $b w(k-2), 9 \leq k \leq 12$. For any of these values White has a dominant move which will result in a gain of -1 points to him. So, any game with board size $n \geq 11$ results in a Black win by 1 point.

Figure 3.2 shows the middle section of the final board for $n \geq 11$, assuming that the left hand side of the board is all Black's territory and the right is White's territory. The diagram also assumes the two players have used 
the optimal strategy described above. After each player has moved $l$ times, Black's next move is in block $b w(k)$, where $k=(n-6 l+2)$. This explains the cyclical repetition of the six final board configurations.

Of course, there are other possible moves which would result in the same final score. A limited number of experiments indicate that if each player makes a dominant move at every turn, the final configuration of the board will be similar to the ones shown in Figure 3.2, though the blocks may be found in a different order. More work is needed to prove this hypothesis.

Table A.1 at the end of the paper shows the left stops for all blocks up to $k=32$. The values of $\mathbf{L S}(d d(k))$ are the score to Black when $n=k$ and both players use their optimal strategy. If either player makes a suboptimal move, the other player can improve her score by responding immediately with a dominant move.

\subsection{Ending the Game}

The definitions of combinatorial game theory assume that the two players must make alternate moves and the last player to have a legal move in the game wins. This does not agree with the rules of a game like Go where a player may pass, the player with the highest number of points for territory and prisoners wins the game and ties are possible. Nevertheless, the theory has been used to successfully analyze the game of Go [3].

Mathematical Go is a version of the game which accommodates these differences. It is based largely on the ancient Chinese version of Go, in which the players continued to play by filling in as much as possible of their own 
territory and the player with the greatest number of stones on the board was the winner. In the modern version, the players stop when both agree that neither can gain further points by making another move. In mathematical Go, the score is calculated as if the players had continued to play until every vacant node, except those in seki, is covered by a stone.

These extra moves are purely imaginary, and are for scoring purposes only. For every pass made before the end of the game, a player gives up a stone to her opponent as a prisoner. Once both players agree that neither can gain from further play, they take turns placing stones on the remaining vacant nodes inside dame and established territory. Any prisoners they have captured are placed in opponent's territory and they use their own stones inside their own territory. In this way, the player with the most points is the last person to play. The vacant nodes in seki cannot be used. In Onedimensional Go these are the blocks $b w(1)$.

Combinatorial game theory does not allow ties and assumes a win for the last player to move. In One-dimensional Go, the game only ends in a score of 0 for very small boards of size $n=1,2$ or 5 . By the rules of Mathematical Go these would all be wins by White since he is the last player to move (or pass). $d d(6)=*$ is considered a win by Black. All these cases would be a tie by the rules of Go. The configuration of the final board for small values of $n$ are shown in Figure 3.1.

In the modern game of Go, Black pays a penalty called a komi for the privilege of moving first. In international matches, the value assigned to the komi varies according to the tournament director. In One-dimensional Go, Black clearly has an advantage of 1 point, so it would be logical to use a 
value of -1 for the $k o m i$ for board sizes of $n \geq 9$. In general, giving a komi equal to - $\mathbf{L S}(d d(n))$ would ensure a final score of zero when both players employ an optimal strategy.

The assumption that prevents ties can be accommodated by assigning a non-integer value to the komi. In One-dimensional Go, a komi of $\frac{1}{2}$ gives Black an overall advantage, whereas a komi of $1 \frac{1}{2}$ gives White the advantage. None of this makes any difference to the players' optimal strategy, and there is no reason for disallowing ties in One-dimensional Go. 


\section{Chapter 4}

\section{Conclusion}

Using combinatorial game theory to analyze the game of One-dimensional Go, we find that Black can force a win for every board size $n \geq 9$. We have also described a strategy by which Black can achieve this result. White can keep his loss down to one point by using a similar strategy. This is not the only possible strategy for each player, and more work may be done to explore alternative ways of reaching this end.

The conventions used in playing a one-dimensional version of the game of Go need not be those described in Chapter 1. The game of Go has a number of dialects which determine how the final board is scored and how to respond to a $k o$.

In several dialects, including the modern Chinese version of the game, points are scored for area rather than territory. This means the players score one extra point for each of their stones on the board at the end of the game. When Black is the last player to move, she places one more stone on the board than White does and will score one more point. Passing before the 


\begin{tabular}{|c|c|c|}
\hline & \multicolumn{2}{|c|}{ Final score to Black } \\
\hline$n$ & Rules used in the paper & Modern Chinese rules \\
\hline 1 & 0 & 0 \\
\hline 2 & 0 & 0 \\
\hline 3 & 2 & 3 \\
\hline 4 & 3 & 4 \\
\hline 5 & 0 & 0 \\
\hline 6 & 0 & 1 \\
\hline 7 & 1 & 2 \\
\hline 8 & 2 & 3 \\
\hline 9 & 1 & 1 \\
\hline$n \geq 10, n$ even & 1 & 2 \\
\hline$n \geq 11, n$ odd & 1 & 1 \\
\hline
\end{tabular}

Table 4.1: Comparing different rule sets.

end of the game costs that player one point for every pass. Also, a stone played in dame gains the player another point. If this rule set is used in our game of One-dimensional Go the final score is also affected.

We have previously scen Figure 3.1 and Figure 3.2 showing the final configuration of the board. Black has one extra stone on the board when $n=3,4,7$ or 8 , and when $n=0,4$ or $5 \bmod 6$ for $n \geq 10$. Black gains one extra point for placing a stone in $b w(2)$ when $n=6$ and when $n=3$ $\bmod 6$. White gains one extra point for placing a stone in $b w(2)$ when $n=0$ $\bmod 6$. Putting this together, for $n \geq 10$ Black gains one more point for all 
odd values of $n$. The figures assume that both players have used the optimal strategy described in section 3.2, and neither have passed before the end of the game. The final scores for One-dimensional Go using our rules and the Chinese rules are compared in Table 4.1. When the game results in a Black win under our rules, Black also wins under the Chinese rules.

In the classic game of Go, the rules which dictate how to respond to a ko also vary between different dialects of the game. These variations may affect the strategy used by the players as well as the final score.

Some versions make a distinction between global and local kos. In our version of the game only a global ko prevents a recapture. When a player is not permitted to play in either type of $k o$, it means that once a stone is captured in a $k o$ the player may never recapture even after the configuration of the rest of the board is changed. This rule would make a significant difference to the optimal strategy for One-dimensional Go. Creating the block $b w(0)$ would always be suboptimal and creating $b d(2)$ would not be a reversible move, and hence neither would $b d(4)$ or $b d(6)$. As a result the outcome of the game could be affected, especially on small boards. For instance, $d d(5)^{L}=4$ under these rules.

Alternatively, there are versions of Go which do not prohibit a recapture in a $k o$ at all. Under some rule sets a ko ends the game in a draw [2]. By the Japanese rules a ko means the game is replayed. Another way of dealing with a ko in mathematical Go is to employ a technique known as chilling, which assigns a value to the $k o$. With a two-dimensional board, there are many different configurations which involve a $k o$, and the value of each can be calculated [3]. A similar method might be employed in One-dimensional 

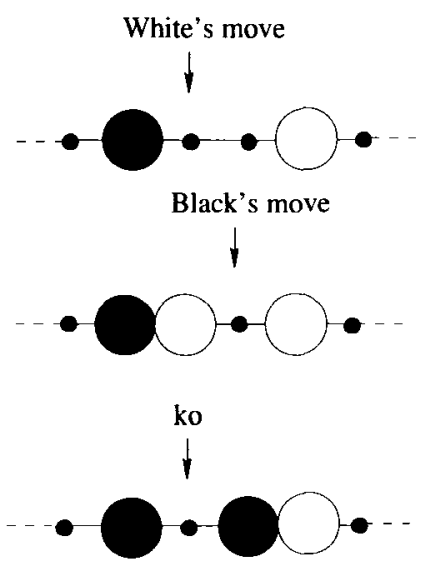

Figure 4.1: A ko threat in One-dimensional Go.

Go. Again, allowing play in a ko may affect the players' optimal strategy as well as the final score of the game.

The analysis described in this paper uses a simplified evaluation of the ko. Figure 4.1 shows that White can initiate a ko threat in a block $b w(2)$ by placing a stone next to the black stone. If Black captures the white stone in a ko White can not recapture immediately. However, White can take advantage of the position by initiating a second attack inside black territory, in a block $b b(2)$ as shown in Figure 1.3. Black now has two stones under attack in two different blocks and whatever her next move, she is likely to lose at least one stone. Since Black has made a capture, White would need to capture at least two black stones to justify such a move. However, this example shows that the analysis of moves involving a ko does not account for the full set of options open to both players. A complete solution to the game of One-dimensional Go would need to address this issue.

This strategic use of the $k o$ can be used by either player in a block $b w(2)$. 
However, it should be noted that when both players use the optimal strategy described in Chapter 3, neither will create a block $b w(2)$ in the middle of a partly played board. Furthermore, if Black does create such a block she also creates a block $b b(k)$, and, for $k \geq 3$, White can gain an advantage by playing into the middle of this block. Similarly, if White creates $b w(2)$ during play he also creates a block $w w(k)$ into which Black can play immediately and gain an advantage.

To find a full solution for One-dimensional Go, the values used for all reversible moves need more rigorous justification than those shown in this paper, especially when a $k o$ is involved. It may be noted that when both players use the optimal strategy described in Chapter 3, neither makes any captures. This would suggest that on a one-dimensional board, a player gains no advantage by initiating an attack on her opponent's stones. This hypothesis also needs further investigation.

It is interesting to observe that both players begin by securing territory near an end of the board when using the optimal strategy described in the paper. A successful strategy for playing the game of Go itself also involves securing territory near a boundary of the board. This raises the question of what might be an optimal strategy on a board without boundaries.

Consider the one-dimensional case, with $n \geq 3$ nodes arranged on the circumference of a circle. Black has no strategy for her opening move, since all the nodes are identical. When $n$ is even, White has a winning strategy by placing a stone in the antipodal position to Black's last move at every turn. White's first move creates $b w\left(\frac{n}{2}-1\right)+b w\left(\frac{n}{2}-1\right)=0$ and White has the final move. 
When $n$ is odd, Black has a winning strategy. White's first move creates $b w(m)$ and $b w(n-m-2)$, where $m<n-m-2$. Black can now create another block $b w(m)$ so that the total value of the board is $b w(m)+b w(m)+$ $b b(n-2 m-3)=0+b b(n-2 m-3)$. Since $\mathrm{n}$ is odd, $(n-2 m-3)$ is even. The right stop of $b b(k)=1$ for all even values of $k$ except for $k=4$ and $k=10$, and for both these cases $b b(k)=0$, with Black playing the last move.

So, it would appear that the boundaries are essential for an interesting game with more than one optimal strategy for each player. This may also be true for the game of Go, though this hypothesis has not been proved. Further research may resolve these unanswered questions. 


\section{Appendix A}

\section{Tables}

The tables show only the left stops. The right stops for the same blocks are

$$
\begin{aligned}
& \mathbf{R S}(b w(k))=-\mathbf{L S}(b w(k)) \\
& \mathbf{R S}(b b(k))=-\mathbf{L S}(w w(k)) \\
& \mathbf{R S}(w w(k))=-\mathbf{L S}(b b(k)) \\
& \mathbf{R S}(b d(k))=-\mathbf{L S}(w d(k)) \\
& \mathbf{R S}(w d(k))=-\mathbf{L S}(b d(k))
\end{aligned}
$$




\begin{tabular}{|r|r|r|r|r|r|r|}
\hline$k$ & $b w(k)$ & $b b(k)$ & $w w(k)$ & $b d(k)$ & $w d(k)$ & $d d(k)$ \\
\hline 1 & 0 & 1 & -1 & 1 & -1 & 0 \\
\hline 2 & 0 & 2 & -2 & 1 & 3 & 0 \\
\hline 3 & 1 & 2 & 0 & 2 & 1 & 2 \\
\hline 4 & 2 & 3 & 0 & 3 & 1 & 3 \\
\hline 5 & 2 & 4 & 0 & 1 & 0 & 0 \\
\hline 6 & 1 & 2 & -1 & 1 & 0 & 0 \\
\hline 7 & 1 & 2 & 0 & 1 & 0 & 1 \\
\hline 8 & 1 & 2 & 1 & 2 & 0 & 2 \\
\hline 9 & 1 & 3 & 0 & 1 & 0 & 1 \\
\hline 10 & 1 & 2 & 0 & 2 & 0 & 1 \\
\hline 11 & 1 & 3 & 0 & 2 & 0 & 1 \\
\hline 12 & 1 & 2 & -1 & 2 & 0 & 1 \\
\hline 13 & 1 & 3 & 0 & 2 & 0 & 1 \\
\hline 14 & 1 & 3 & -1 & 2 & 0 & 1 \\
\hline 15 & 1 & 3 & 0 & 2 & 0 & 1 \\
\hline 16 & 1 & 2 & -1 & 2 & 0 & 1 \\
\hline 17 & 1 & 3 & 0 & 2 & 0 & 1 \\
\hline 18 & 1 & 2 & 1 & 2 & 0 & 1 \\
\hline 19 & 1 & 3 & 0 & 2 & 0 & 1 \\
\hline 20 & 1 & 2 & -1 & 2 & 0 & 1 \\
\hline 21 & 1 & 3 & 0 & 2 & 0 & 1 \\
\hline 22 & 1 & 3 & -1 & 2 & 0 & 1 \\
\hline 23 & 1 & 3 & 0 & 2 & 0 & 1 \\
\hline 24 & 1 & 3 & -1 & 2 & 0 & 1 \\
\hline 25 & 1 & 3 & 0 & 2 & 0 & 1 \\
\hline 26 & 1 & 2 & -1 & 2 & 0 & 1 \\
\hline 27 & 1 & 3 & 0 & 2 & 0 & 1 \\
\hline 28 & 1 & 3 & 1 & 2 & 0 & 1 \\
\hline 29 & 1 & 3 & 0 & 2 & 0 & 1 \\
\hline 30 & 1 & 3 & -1 & 2 & 0 & 1 \\
\hline 31 & 1 & 3 & 0 & 2 & 0 & 1 \\
\hline 32 & 1 & 2 & -1 & 2 & 0 & 1 \\
\hline
\end{tabular}

Table A.1: Left stops for given blocks. 


\begin{tabular}{|c|c|c|c|c|c|c|c|c|c|c|c|c|c|c|c|c|c|c|}
\hline \multirow[b]{2}{*}{$k$} & \multirow[b]{2}{*}{$\mathbf{L S}(b w(k))$} & \multicolumn{17}{|c|}{$\mathbf{R S}(b b(m)+b w(k-m-1)): m=$} \\
\hline & & 0 & 1 & 2 & 3 & 4 & 5 & 6 & 7 & 8 & 9 & 10 & 11 & 12 & 13 & 14 & 15 & 16 \\
\hline 3 & 1 & 0 & 1 & & & & & & & & & & & & & & & \\
\hline 4 & 2 & -1 & 1 & $\mathbf{2}$ & & & & & & & & & & & & & & \\
\hline 5 & 2 & -2 & 0 & 2 & 0 & & & & & & & & & & & & & \\
\hline 6 & 1 & -2 & -1 & 1 & 0 & 0 & & & & & & & & & & & & \\
\hline 7 & 1 & -1 & -1 & 0 & 1 & 0 & 0 & & & & & & & & & & & \\
\hline 8 & 1 & -1 & 0 & 0 & 0 & $\mathbf{1}$ & 0 & 1 & & & & & & & & & & \\
\hline 9 & 1 & -1 & 0 & 1 & 0 & 1 & 0 & 1 & 0 & & & & & & & & & \\
\hline 10 & 1 & -1 & 0 & 1 & -1 & 1 & 1 & 1 & 0 & 1 & & & & & & & & \\
\hline 11 & 1 & -1 & 0 & 1 & 0 & 0 & 1 & 0 & 1 & 1 & 0 & & & & & & & \\
\hline 12 & 1 & -1 & 0 & 1 & 0 & 0 & 1 & 0 & 0 & 1 & 0 & 0 & & & & & & \\
\hline 13 & 1 & -1 & 0 & 1 & 0 & 0 & 0 & 0 & 0 & 0 & 1 & 0 & 0 & & & & & \\
\hline 14 & 1 & -1 & 0 & 1 & 0 & 1 & 0 & 1 & 1 & 0 & 1 & 1 & 0 & 1 & & & & \\
\hline 15 & 1 & -1 & 0 & 1 & 0 & 0 & 1 & 1 & 1 & 0 & 1 & 0 & 0 & 1 & 0 & & & \\
\hline 16 & 1 & -1 & 0 & 1 & 0 & 0 & 1 & 0 & 1 & 1 & 0 & 0 & 1 & 0 & 0 & 1 & & \\
\hline 17 & 1 & -1 & 0 & 1 & 0 & 0 & 1 & 0 & 0 & 1 & 0 & 1 & 1 & 0 & 1 & 1 & 0 & \\
\hline 18 & 1 & -1 & 0 & 1 & 0 & 1 & 1 & 0 & 0 & 1 & 0 & 0 & 1 & 0 & 1 & 1 & 0 & 1 \\
\hline
\end{tabular}

Table A.2: Dominant Black moves for blocks $b w(k)$. 


\begin{tabular}{|c|c|c|c|c|c|c|c|c|c|c|c|c|c|c|c|c|c|}
\hline \multirow[b]{2}{*}{$k$} & \multirow[b]{2}{*}{$\mathbf{L S}(b b(k))$} & \multicolumn{16}{|c|}{$\mathbf{R S}(b b(m)+b b(k-m-1)): m=$} \\
\hline & & 0 & 1 & 2 & 3 & 4 & 5 & 6 & 7 & 8 & 9 & 10 & 11 & 12 & 13 & 14 & 15 \\
\hline 3 & 2 & 2 & 2 & & & & & & & & & & & & & & \\
\hline 4 & 3 & 0 & 3 & & & & & & & & & & & & & & \\
\hline 5 & 4 & 0 & 1 & 4 & & & & & & & & & & & & & \\
\hline 6 & 2 & 0 & 1 & 2 & & & & & & & & & & & & & \\
\hline 7 & 2 & 0 & 1 & 2 & 2 & & & & & & & & & & & & \\
\hline 8 & 2 & 0 & 2 & $\mathbf{2}$ & 2 & & & & & & & & & & & & \\
\hline 9 & 3 & 0 & 1 & 3 & 1 & 3 & & & & & & & & & & & \\
\hline 10 & 2 & 0 & 2 & 2 & 1 & 2 & & & & & & & & & & & \\
\hline 11 & 3 & 0 & 1 & 3 & 2 & 2 & 3 & & & & & & & & & & \\
\hline 12 & 2 & 0 & 1 & 2 & 2 & 2 & 2 & & & & & & & & & & \\
\hline 13 & 3 & 0 & 1 & 2 & 2 & 2 & 2 & 3 & & & & & & & & & \\
\hline 14 & 3 & 0 & 2 & 2 & 2 & 3 & 2 & 2 & & & & & & & & & \\
\hline 15 & 3 & 0 & 1 & $\mathbf{3}$ & 1 & 2 & 2 & 2 & 2 & & & & & & & & \\
\hline 16 & 2 & 0 & 2 & 2 & 1 & 2 & 2 & 2 & 2 & & & & & & & & \\
\hline 17 & 3 & 0 & 1 & $\mathbf{3}$ & 2 & 2 & 3 & 2 & 2 & 3 & & & & & & & \\
\hline 18 & 2 & 0 & 2 & 2 & 2 & 2 & 2 & 2 & 2 & 2 & & & & & & & \\
\hline 19 & 3 & 0 & 1 & 3 & 2 & 2 & 2 & 3 & 2 & 2 & 3 & & & & & & \\
\hline 20 & 2 & 0 & 2 & 2 & 2 & 2 & 2 & 2 & 2 & 2 & 2 & & & & & & \\
\hline 21 & 3 & 0 & 1 & $\mathbf{3}$ & 2 & 2 & 3 & 2 & 2 & 2 & 2 & 2 & & & & & \\
\hline 22 & 3 & 0 & 2 & 2 & 2 & 2 & 2 & 2 & 3 & 2 & 2 & 2 & & & & & \\
\hline 23 & 3 & 0 & 1 & 3 & 2 & 2 & 3 & 2 & 2 & 3 & 2 & 2 & 3 & & & & \\
\hline 24 & 3 & 0 & 2 & 2 & 2 & 2 & 2 & 2 & 2 & 2 & 3 & 2 & 2 & & & & \\
\hline 25 & 3 & 0 & 1 & 3 & 2 & 2 & 3 & 3 & 2 & 2 & 3 & 2 & 2 & 3 & & & \\
\hline 26 & 2 & 0 & 2 & 2 & 2 & 2 & 2 & 2 & 2 & 2 & 2 & 2 & 2 & 2 & & & \\
\hline 27 & 3 & 0 & 1 & $\mathbf{3}$ & 2 & 2 & 3 & 2 & 2 & 3 & 2 & 2 & 2 & 2 & 2 & & \\
\hline 28 & 3 & 0 & 2 & 2 & 2 & 2 & $\mathbf{3}$ & 2 & 2 & 2 & 2 & 2 & 2 & 2 & 2 & & \\
\hline 29 & 3 & 0 & 1 & 3 & 2 & 2 & 3 & 2 & 2 & 3 & 2 & 2 & 3 & 3 & 2 & 3 & \\
\hline 30 & 3 & 0 & 2 & 2 & 2 & 2 & 3 & 2 & 3 & 2 & 2 & 2 & 2 & 2 & 2 & 3 & \\
\hline 31 & 3 & 0 & 1 & $\mathbf{3}$ & 2 & 2 & 3 & 2 & 2 & 3 & 3 & 2 & 2 & 3 & 3 & 2 & 3 \\
\hline 32 & 2 & 0 & 2 & 2 & 2 & 2 & 2 & 2 & 2 & 2 & 2 & 2 & 2 & 2 & 2 & 2 & 2 \\
\hline
\end{tabular}

Table A.3: Dominant Black moves for blocks $b b(k)$. 


\begin{tabular}{|c|c|c|c|c|c|c|c|c|c|c|c|c|}
\hline \multirow[b]{2}{*}{$k$} & \multirow[b]{2}{*}{$\mathbf{L S}(w w(k))$} & \multicolumn{6}{|c|}{$\mathbf{R S}(b u(m)+b w(k$} & \multicolumn{5}{|c|}{ 1)): $m=$} \\
\hline & & 1 & 2 & 3 & 4 & 5 & 6 & 7 & 8 & 9 & 10 & 11 \\
\hline 3 & 0 & o & & & & & & & & & & \\
\hline 4 & 0 & o & & & & & & & & & & \\
\hline 5 & 0 & -1 & 0 & & & & & & & & & \\
\hline 6 & -1 & -2 & -1 & & & & & & & & & \\
\hline 7 & 0 & -2 & -2 & $\mathbf{o}$ & & & & & & & & \\
\hline 8 & -1 & -1 & -2 & -1 & & & & & & & & \\
\hline 9 & 0 & -1 & -1 & -1 & 0 & & & & & & & \\
\hline 10 & 0 & -1 & -1 & -2 & $\mathbf{o}$ & & & & & & & \\
\hline 11 & 0 & 1 & -1 & $\cdots 1$ & 1 & o & & & & & & \\
\hline 12 & -1 & -1 & -1 & -1 & -2 & -1 & & & & & & \\
\hline 13 & 0 & -1 & -1 & -1 & -2 & -2 & $\mathbf{0}$ & & & & & \\
\hline 14 & -1 & -1 & -1 & -1 & -1 & -2 & -1 & & & & & \\
\hline 15 & 0 & -1 & -1 & -1 & -1 & -1 & -1 & o & & & & \\
\hline 16 & -1 & -1 & -1 & -1 & -1 & -1 & -2 & -1 & & & & \\
\hline 17 & 0 & -1 & -1 & -1 & -1 & -1 & -1 & -1 & 0 & & & \\
\hline 18 & -1 & -1 & -1 & -1 & -1 & -1 & -1 & -2 & -1 & & & \\
\hline 19 & 0 & -1 & -1 & -1 & -1 & -1 & -1 & -1 & -1 & 0 & & \\
\hline 20 & -1 & -1 & -1 & -1 & -1 & -1 & -1 & -1 & -2 & -1 & & \\
\hline 21 & 0 & -1 & 1 & 1 & 1 & 1 & 1 & 1 & 1 & 1 & o & \\
\hline 22 & 1 & 1 & 1 & 1 & 1 & 1 & 1 & 1 & 1 & 2 & -1 & \\
\hline 23 & 0 & -1 & -1 & -1 & -1 & -1 & -1 & -1 & -1 & -1 & -1 & 0 \\
\hline 24 & -1 & -1 & -1 & -1 & -1 & -1 & -1 & -1 & -1 & -1 & -2 & -1 \\
\hline
\end{tabular}

Table A.4: Dominant Black moves for blocks $w w(k)$. 


\begin{tabular}{|c|c|r|r|r|r|r|r|r|r|r|r|r|}
\hline & & \multicolumn{10}{|c|}{$\mathbf{R S}(b d(m)+b b(k-m-1)): m=$} \\
\hline$k$ & $\mathbf{L S}(b d(k))$ & 0 & 1 & 2 & 3 & 4 & 5 & 6 & 7 & 8 & 9 & 10 \\
\hline 3 & 2 & -2 & $\mathbf{2}$ & -5 & & & & & & & & \\
\hline 4 & 3 & -2 & $\mathbf{3}$ & -5 & -1 & & & & & & & \\
\hline 5 & 1 & -2 & 1 & -3 & 0 & -3 & & & & & & \\
\hline 6 & 1 & -2 & 1 & -1 & 1 & -3 & 0 & & & & & \\
\hline 7 & 1 & -1 & 1 & 0 & 1 & -2 & 1 & 0 & & & & \\
\hline 8 & 2 & 2 & 2 & 0 & 2 & 2 & 2 & 1 & 0 & & & \\
\hline 9 & 2 & -1 & 1 & -1 & 1 & -1 & 0 & 2 & 1 & 0 & & \\
\hline 10 & 2 & -2 & 2 & -1 & 1 & 0 & 1 & 1 & 2 & 1 & 0 & \\
\hline 11 & 2 & -2 & 1 & -1 & 1 & -2 & 1 & 1 & 1 & 2 & 1 & 0 \\
\hline 12 & 2 & -2 & 1 & 0 & 1 & -2 & 2 & 1 & 1 & 1 & 2 & 1 \\
\hline 13 & 2 & -1 & 1 & -1 & 2 & -2 & 1 & 2 & 1 & 2 & 1 & 2 \\
\hline 14 & 2 & -2 & 2 & 0 & 1 & -1 & 1 & 1 & 1 & 1 & 1 & 1 \\
\hline 15 & 2 & -1 & 1 & -1 & 1 & -2 & 1 & 2 & 1 & 1 & 2 & 1 \\
\hline 16 & 2 & -2 & 2 & 0 & 1 & -1 & 1 & 1 & 2 & 1 & 1 & 2 \\
\hline 17 & 2 & -1 & 1 & 0 & 1 & -2 & 1 & 1 & 1 & 2 & 1 & 2 \\
\hline 18 & 2 & -2 & 2 & 0 & 1 & 1 & 2 & 1 & 1 & 2 & 1 & 1 \\
\hline 19 & 2 & -1 & 1 & 1 & 1 & 1 & 1 & 2 & 1 & 2 & 2 & 1 \\
\hline 20 & 2 & -2 & 2 & 0 & 1 & -1 & 1 & 1 & 1 & 1 & 2 & 1 \\
\hline 21 & 2 & -1 & 1 & -1 & 1 & -2 & 1 & 2 & 1 & 1 & 2 & 2 \\
\hline 22 & 2 & -2 & 2 & 0 & 1 & -1 & 1 & 1 & 2 & 2 & 1 & 2 \\
\hline 23 & 2 & -1 & 1 & -1 & 1 & -2 & 1 & 2 & 1 & 2 & 1 & 2 \\
\hline 24 & 2 & -2 & 2 & 0 & 1 & -1 & 2 & 1 & 1 & 2 & 2 & 1 \\
\hline
\end{tabular}

\begin{tabular}{|r|c|r|r|r|r|r|r|r|r|r|r|r|r|}
\hline & \multicolumn{10}{|c|}{} & \multicolumn{10}{|c|}{ RS $(b d(m)+h b(k-m-1)): m=$} \\
\hline$k$ & $\mathbf{L S}(b d(k))$ & 11 & 12 & 13 & 14 & 15 & 16 & 17 & 18 & 19 & 20 & 21 & 22 \\
\hline 12 & 2 & 0 & & & & & & & & & & & \\
\hline 13 & 2 & 1 & 0 & & & & & & & & & & \\
\hline 14 & 2 & 2 & 1 & 0 & & & & & & & & & \\
\hline 15 & 2 & 1 & 2 & 1 & 0 & & & & & & & & \\
\hline 16 & 2 & 1 & 1 & 2 & 1 & 0 & & & & & & & \\
\hline 17 & 2 & 2 & 1 & 1 & 2 & 1 & 0 & & & & & & \\
\hline 18 & 2 & 2 & 2 & 2 & 1 & 2 & 1 & 0 & & & & & \\
\hline 19 & 2 & 2 & 1 & 2 & 1 & 1 & 2 & 1 & 0 & & & & \\
\hline 20 & 2 & 2 & 2 & 1 & 2 & 1 & 1 & 2 & 1 & 0 & & & \\
\hline 21 & 2 & 1 & 2 & 1 & 1 & 2 & 1 & 1 & 2 & 1 & 0 & & \\
\hline 22 & 2 & 1 & 2 & 2 & 1 & 1 & 2 & 1 & 1 & 2 & 1 & 0 & \\
\hline 23 & 2 & 2 & 1 & 2 & 2 & 1 & 2 & 2 & 1 & 1 & 2 & 1 & 0 \\
\hline 24 & 2 & 2 & 1 & 2 & 2 & 2 & 2 & 1 & 2 & 2 & 1 & 2 & 1 \\
\hline
\end{tabular}

Table A.5: Dominant Black moves for blocks $b d(k)$. 


\begin{tabular}{|c|c|c|}
\hline$k$ & $b w(k)$ & $b b(k)$ \\
\hline 0 & & 0 \\
\hline 1 & 0 & 1 \\
\hline 2 & $*$ & 2 \\
\hline 3 & \pm 1 & $\{2 \mid 0\}$ \\
\hline 4 & \pm 2 & $\{3 \mid *\}$ \\
\hline 5 & $\pm(2 *)$ & $\{4 \mid 0, \pm 1\}$ \\
\hline 6 & $\pm\{3 \mid 1\}$ & $\{4|2| \mid \pm(1 *)\}$ \\
\hline 7 & $\pm(1,\{4 \mid 0\})$ & $\{5|2 *| \mid 0\}$ \\
\hline 8 & $\pm(\{4|2| \mid( \pm(1 *),\{4 * \mid *\})$ & $\{6|2,\{3 \mid 1\}| \mid 1\}$ \\
\hline 9 & $\pm(\{5|2 *| \mid 0\},\{5|3| \mid \pm 1\})$ & $\{6|4||3 *| 1 *|| \mid 0\}$ \\
\hline 10 & $\pm(\{3 \mid *\},\{3,6 \mid 2\} \mid 1, \pm 2\})$ & $\{7|4 *||2||| *\}$ \\
\hline 11 & & $\{8|4,\{5 \mid 3\}||3||| 0, \pm 1\}$ \\
\hline 12 & & $\{8|6||5 *| 3 *|||2||| \mid \pm(1 *)\}$ \\
\hline
\end{tabular}

Table A.6: Some values for blocks $b w(k)$ and $b b(k)$. 


\begin{tabular}{|c|c|c|}
\hline$k$ & $b d(k)$ & $d d(k)$ \\
\hline 0 & -2 & 0 \\
\hline 1 & 1 & 0 \\
\hline 2 & $\{1 \mid-3\}$ & 0 \\
\hline 3 & $\{2 \mid-1\}$ & \pm 2 \\
\hline 4 & $\{3 \mid-4,\{-1 \mid-4\}\}$ & \pm 3 \\
\hline 5 & $\{3|1||0|-2\}$ & 0 \\
\hline 6 & $\{\{4 \mid 1\},\{4 \mid 1 *\} \mid *\}$ & $*$ \\
\hline 7 & $\{1,\{5 \mid 1,\{2 \mid 0\}\} \mid 0,\{1 * \mid-3 *\}\}$ & \pm 1 \\
\hline 8 & & $\pm\{\{5 \mid 2\},\{5 \mid 2 *\} \mid \pm 2,\{2 * \mid-1 *\}\}$ \\
\hline 9 & & $\pm\{2,\{6 \mid 2,\{3 \mid 1\}\} \mid 1, \pm(2 *)\}$ \\
\hline
\end{tabular}

Table A.7: Some values for blocks $b d(k)$ and $d d(k)$. 


\section{Appendix B}

\section{Recursion}

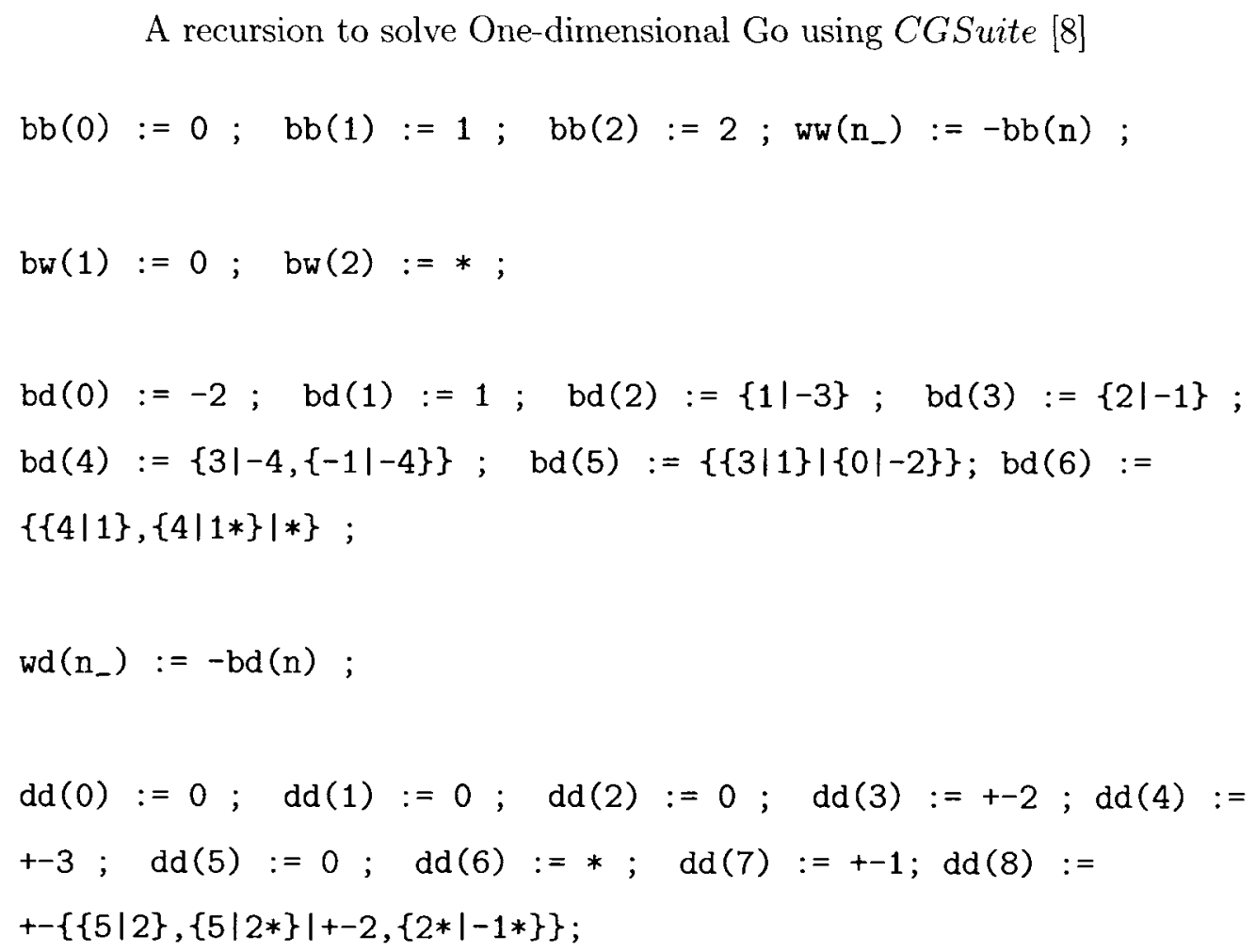


$\mathrm{bw}\left(\mathrm{n}_{-}\right):=\{1 \mathrm{bw}(\mathrm{n}) \mid \operatorname{rbw}(\mathrm{n})\} ; \operatorname{rbw}\left(\mathrm{n}_{-}\right):=\operatorname{seq}(-1.1 \mathrm{bw}(\mathrm{n})[\mathrm{k}]$, $k=1$. Length $(1 \mathrm{bw}(n)))$;

lbw $:=\operatorname{proc}(n)$ local $k$, list ; option remember ; if $n==1$ then list $:=[]$; elif $\mathrm{n}==2$ then list $:=[0]$; else list $:=[]$; Append(list, $\operatorname{seq}(b b(k)+b w(n-k-1), k=0 \ldots n-2)) ; A p p e n d(1$ ist, $\operatorname{seq}(-2+1 b w(n-2)[k]$, $k=1$. Length $(\operatorname{lbw}(n-2)))) ; f i$; list end ;

$\mathrm{bb}\left(\mathrm{n}_{-}\right):=\{\mathrm{lbb}(\mathrm{n}) \mid \operatorname{rbb}(\mathrm{n})\} ; \mathrm{lbb}\left(\mathrm{n}_{-}\right):=\operatorname{seq}(\mathrm{bb}(\mathrm{k})+\mathrm{bb}(\mathrm{n}-\mathrm{k}-1)$, $\mathrm{k}=0 . . \mathrm{n}-1)$;

$\mathrm{rbb}:=\operatorname{proc}(\mathrm{n})$ local $\mathrm{k}$, list ; option remember; if $\mathrm{n}==1$ then list $:=[]$; elif $\mathrm{n}==2$ then list $:=[0]$; else list $:=[]$; Append(list, $\operatorname{seq}(b w(k)+b w(n-k-1), k=1 \ldots n-2)) ; \operatorname{Append}(1$ ist, $\operatorname{seq}(2+r b b(n-2)[k]$, $k=1$. Length $(\operatorname{rbb}(n-2)))) ; f i$; list end ;

$\operatorname{bd}\left(n_{-}\right):=\{\operatorname{lbd}(n) \mid \operatorname{rbd}(n)\} ;$

lbd $:=\operatorname{proc}(n)$ local $k$, list ; option remember; if $n==0$ then list $:=[]$; elif $\mathrm{n}==1$ then list $:=[0]$; elif $\mathrm{n}==2$ then list $:=[1]$; elif $\mathrm{n}==3$ then list $:=[2]$; elif $\mathrm{n}==4$ then list $:=[3]$; else list $:=[]$; Append (list, $\operatorname{seq}(-2+1 b w(n-2)[k], k=1 \ldots \operatorname{Length}(1 b w(n-2)))$ ); Add (list, $1+b b(n-2))$; Append(list, $\operatorname{seq}(-3+1 b w(n-4)[k]$, 


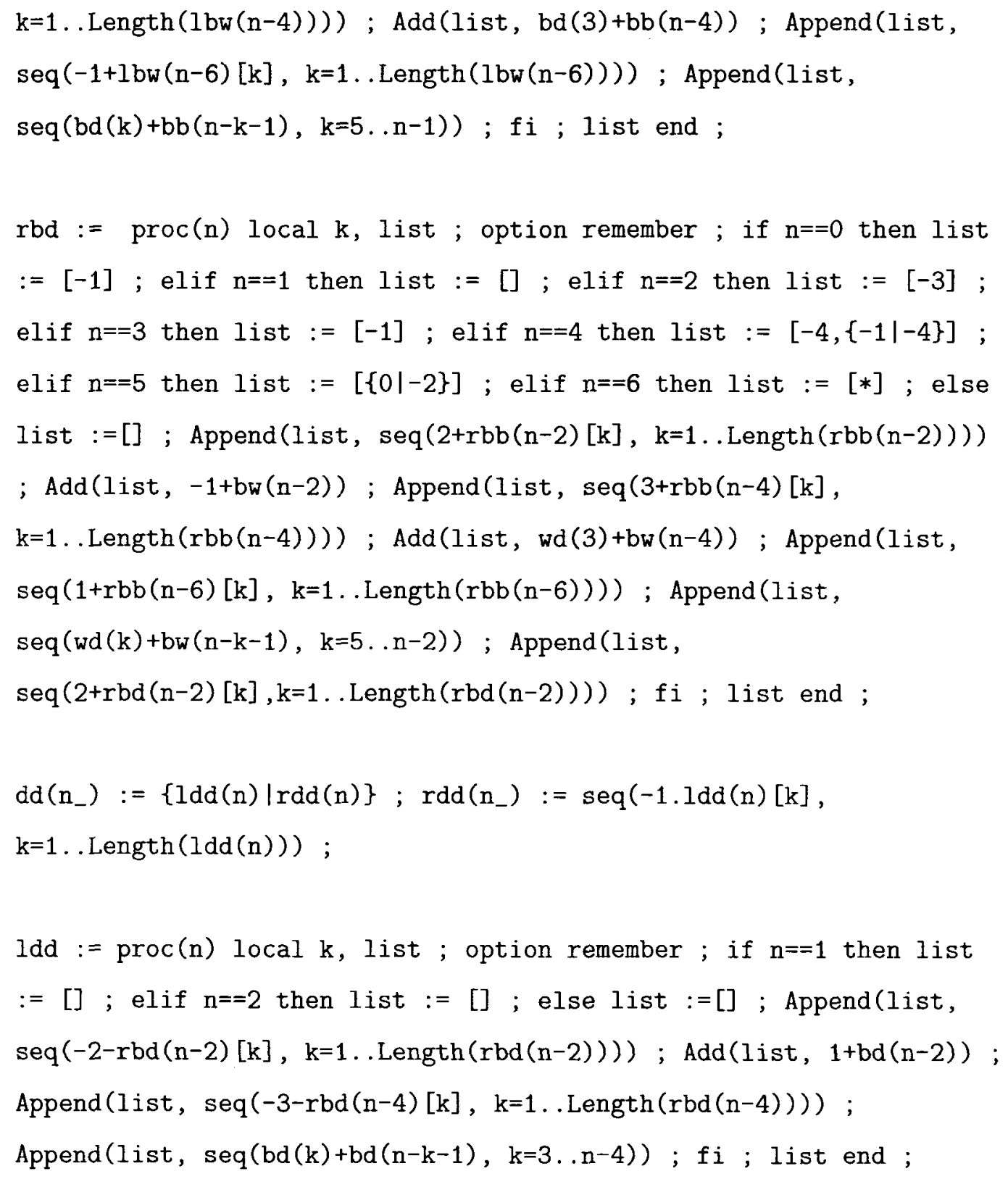




\section{Bibliography}

[1] Michael Albert, Richard Nowakowski and David Wolfe. Lessons in Play: an Introduction to the Combinatorial Theory of Games. A.K. Peters Ltd., Natick, Massachusetts, 2007.

[2] Elwyn R. Berlekamp. "Introductory Overview of Mathematical Go Endgames". Proceedings of Symposia in Applied Mathematics vol. 43, Combinatorial Games, pp. 73-100. American Mathematical Society, 1991.

[3] Elwyn Berlekamp and David Wolfe. Mathematical Go: Chilling Gets the Last Point. A.K. Peters Ltd., Natick, Massachusetts, 1994

[4] Elwyn R. Berlekamp, John H. Conway and Richard K. Guy. Winning Ways for Your Mathematical Plays. A.K. Peters Ltd., Natick, Massachusetts, 2nd edition, 2001. First edition pulished in 1982 by Academic Press.

[5] John H. Conway. On Numbers and Games. A.K. Peters Ltd., Natick, Massachusetts, 2nd edition, 2001. First edition pulished in 1976 by Academic Press. 
[6] Donald E. Knuth. Surreal Numbers. Addison-Wesley, Reading, MA, 1974.

[7] Richard Nowakowski, editor. Games of No Chance: Combinatorial Games at MSRI, 1994. Cambridge University Press, Mathematical Sciences Research Institute Publications 29, 1996.

[8] Aaron N. Siegel. Cgsuite or combinatorial game suite, 2003. Software toolkit for analyzing combinatorial games. http://www.cgsuite.org. 